\title{
Dynamique et estimation du rendement des strates de retour après coupe totale dans la sapinière à bouleau jaune
}

\author{
par Julie Dubois ${ }^{1}$,Jean-Claude Ruel ${ }^{1,2}$, Jean-Gabriel Elie ${ }^{1}$ et Louis Archambault ${ }^{1}$
}

\begin{abstract}
RÉSUMÉ
Un total de 48 placettes issues de coupe totale localisées dans le domaine bioclimatique de la sapinière à bouleau jaune de la province de Québec, Canada, ont été étudiées au moyen d'une analyse dendrochronologique afin d'évaluer l'importance de la composition avant coupe, de l'abondance et de la structure de la régénération après coupe et du type de milieu physique sur la dynamique des peuplements. Les résultats démontrent le lien entre le couvert d'origine et les peuplements de retour. La considération des conditions écologiques apparaît indispensable pour caractériser l'importance de la régénération préétablie et pour décrire l'évolution des coefficients de distribution. Les résultats permettent également de mettre en lumière la pertinence de considérer la structure de la régénération, par le biais de l'indice de diamètre, pour prédire le rendement à 38 ans. Les dommages causés par la tordeuse des bourgeons de l'épinette constituent un facteur déterminant dans l'évolution des peuplements dans le cadre de cette étude et rendent difficile la prédiction du rendement des peuplements régénérés en sapin. Enfin, les résultats mettent en évidence le phénomène d'augmentation des feuillus intolérants à l'ombre et les problèmes de productivité résineuse sur stations riches. La pessières noires se reconstituaient en peuplements de même composition.
\end{abstract}

Mots-clés : peuplements mixtes, succession, inventaires de régénération, rendement, Choristoneura fumiferana

\section{ABSTRACT}

A total of 48 plots originating from clearcuts were studied in the balsam fir-yellow birch ecological domain of the province of Quebec, Canada, using dendrochronology. The aim of the study was to assess the effect of pre-cut stand type, structure and abundance of regeneration after logging, and soil type on stand dynamics. Results indicate a relationship between pre-cut stand type and the composition of second-growth stands. Ecological characteristics must be taken into account in order to estimate the abundance of advance regeneration and predict the evolution of stocking. Results also show that the structure of regeneration, described by a diameter index, can help in predicting yield at age 38 . Devastation by the spruce budworm played a major role in second-growth stand dynamics making it difficult to predict balsam fir yield. The study has also shown an increase in intolerant hardwoods and a low softwood production on rich sites. Black spruce stands came back to black spruce.

Key words: mixedwoods, succession, regeneration survey, yield, Choristoneura fumiferana

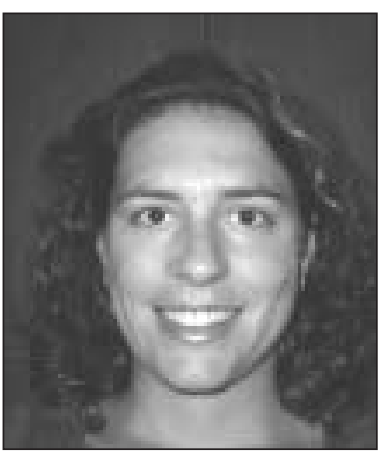

Julie Dubois

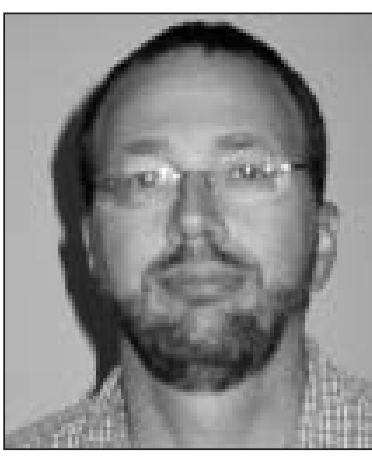

Jean-Claude Ruel

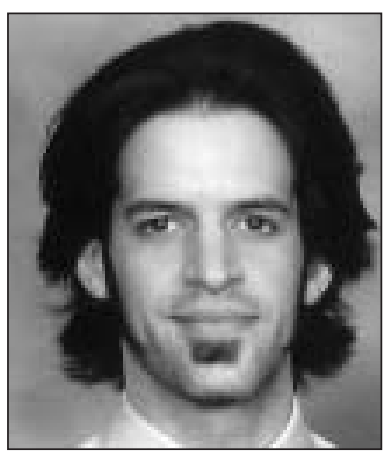

Jean-Gabriel Elie

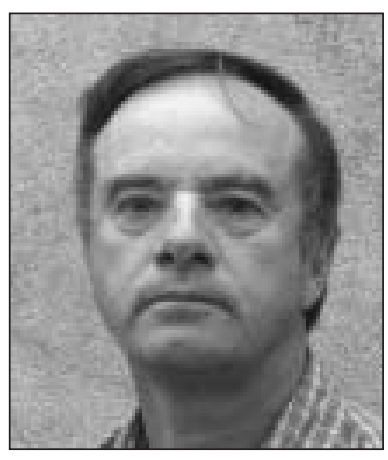

Louis Archambault

\footnotetext{
${ }^{1}$ Département des sciences du bois et de la forêt, Université Laval, Sainte-Foy (Québec) G1K 7P4.

${ }^{2}$ Auteur de correspondance. Courriel : jean-claude.ruel@sbf.ulaval.ca
} 


\section{Introduction}

Le domaine bioclimatique de la sapinière à bouleau jaune revêt une grande importance pour l'industrie forestière québécoise, car il compte des sites parmi les plus productifs au Québec Malgré que de nombreuses études écologiques aient été réalisées au Québec au cours des dernières décennies (Grondin et al. 1996), il demeure que les connaissances sur la composition, les fonctions, la structure et la dynamique des écosystèmes de ce domaine bioclimatique sont encore jugées insuffisantes (Conseil de la recherche forestière du Québec 2002, Prévost et al. 2003). Ces connaissances sont essentielles pour la mise en œuvre de pratiques d'aménagement durable (Bergeron et Harvey 1997, Hunter 1999, Kimmins 2002). De plus, une meilleure compréhension de la dynamique du couvert forestier et des strates de retour après coupe est requise pour l'évaluation de la possibilité forestière des territoires sous aménagement.

En plus des perturbations anthropiques, les écosystèmes du domaine bioclimatique de la sapinière à bouleau jaune peuvent être affectés par différents types de perturbations naturelles (insectes, maladies, chablis, feu) variant en fréquence et en magnitude (Grondin et al. 1996). Ces phénomènes naturels peuvent agir seuls ou en interaction avec les perturbations anthropiques et affecter la dynamique végétale selon des trajectoires difficiles à prévoir. La dynamique des écosystèmes mixtes est particulièrement difficile à évaluer compte tenu des caractéristiques écologiques souvent différentes des nombreuses espèces qui les composent (Oliver et Larson 1990).

L'évolution des peuplements après perturbation est souvent influencée par la nature, la structure et l'abondance de la régénération préétablie (Paquin et Doucet 1992b; Pominville 1993; Pothier et al. 1993, 1995; Riopel 1999). Ces caractéristiques étant en partie dépendantes de la disponibilité des semences, un lien est souvent observé entre la composition du peuplement et les caractéristiques de la régénération préétablie (Morin et Gagnon 1991).

La régénération qui s'ajoute après coupe joue aussi un rôle déterminant sur la composition et le rendement des nouveaux peuplements (Vézina et Linteau 1968; Morin et Gagnon 1991; Paquin et Doucet 1992a; Pominville 1993; Pothier et al. 1993, 1995). La régénération après coupe varie fortement selon les espèces et les stations (Ruel et al. 1998, Symons 1996). En effet, le recrutement après coupe est particulièrement important pour les feuillus intolérants (Frisque et al. 1978, Harvey et Bergeron 1989, Ruel 1990, Ruel 1992a, Sarrasin 1991, Symons 1996). Une des conséquences d'un tel phénomène est que plusieurs peuplements résineux se régénèrent en peuplements mixtes (Frisque et al. 1978, Ruel, 1992b, Pominville 1993, Ruel et Huot 1993, Archambault et al. 1998). D'autres situations présentent un important déclin des feuillus au cours de la période de prématurité, de sorte que la composition à maturité peut différer fortement de celle observée à cinq ou dix ans (Ruel et al. 1998). De plus, l'augmentation possible de la proportion de sapin baumier (Abies balsamea (L.) Mill.) au détriment des épinettes (Hatcher 1960, Fye et Thomas 1963, Vézina et Falardeau 1988) contribue également à complexifier l'exercice de prédiction des strates de retour.

Les espèces de compétition comme l'érable à épis (Acer spicatum Lamb), le framboisier (Rubus idaeus L. var. strigosus
Michx.) et le noisetier à long bec (Corylus cornuta Marsh.) peuvent jouer un rôle important dans la dynamique forestière des sites les plus riches (Bell 1991, Jobidon 1995, Archambault et al. 1998). Ces espèces peuvent envahir les parterres de coupe et influer sur l'évolution des peuplements en plus de causer des diminutions importantes de production.

Les épidémies de la tordeuse des bourgeons de l'épinette (Choristoneura fumiferana (Clem.)) jouent un rôle particulièrement significatif dans le fonctionnement des écosystèmes des domaines bioclimatiques de la sapinière à bouleau jaune et de la sapinière à bouleau blanc (Ghent et al. 1957, Fye et Thomas 1963, Batzer et Popp 1985, Grondin et al. 1996, Kneeshaw et Bergeron 1996). Or plusieurs auteurs ont établi un lien entre les caractéristiques du milieu et la mortalité infligée par la tordeuse des bourgeons de l'épinette (Archambault et al. 1990, Dupont et al. 1991).

Le présent article vise à décrire la dynamique de la végétation après coupe dans le domaine bioclimatique de la sapinière à bouleau jaune de la province de Québec, Canada, et à examiner le lien entre les caractéristiques de la régénération après coupe et le rendement à moyen terme. L'étude applique une approche dendrochronologique à des coupes réalisées autour des années 1960 afin d'obtenir un portrait de l'évolution du couvert forestier sur une période d'environ 40 ans. En particulier, l'étude tente de démontrer l'influence du couvert d'origine et du type écologique sur l'état de la régénération en bas âge, son développement et la composition des strates de retour.

\section{Matériel et Méthodes Territoire d'étude}

Le territoire à l'étude se situe à environ $100 \mathrm{~km}$ à l'ouest de la ville de La Tuque, Québec $\left(47^{\circ} 15^{\prime} \mathrm{N}, 73^{\circ} 45^{\prime} \mathrm{W}\right)$, Canada. Il appartient au sous-domaine bioclimatique de la sapinière à bouleau jaune de l'Ouest, dans la région écologique des Collines du moyen Saint-Maurice (4c) (Gosselin et al. 2001). Les températures et précipitations annuelles moyennes sont respectivement de $1,3^{\circ} \mathrm{C}$ et $1060 \mathrm{~mm}$ (Robitaille et Saucier 1998). Le nombre de degrés-jours de croissance oscille entre 2000 et 2600 unités et la durée de la saison de croissance annuelle varie entre 160 et 170 jours (Gosselin et al. 2001). Le relief est principalement formé de collines aux versants en pente faible ou moyenne et l'altitude moyenne est de 450 mètres. Les tills épais et les dépôts fluvio-glaciaires couvrent d'importantes superficies.

Sur le territoire étudié, la sapinière à bouleau blanc se retrouve fréquemment sur les collines de till, alors que la pessière noire à mousses ou à éricacées couvre les dépôts fluvio-glaciaires caractérisés par un drainage rapide en terrain plat. La sapinière à épinette noire occupe plutôt une position intermédiaire entre ces deux types de végétation. Les superficies à l'étude sont caractérisées par un assemblage de peuplements très diversifié et leur disposition dans l'espace est morcelée en raison des perturbations naturelles et anthropiques qu'elles ont subies.

Selon les registres de la Société de protection des forêts contre les insectes et les maladies (SOPFIM), on recense trois épisodes épidémiques de tordeuse des bourgeons de l'épinette dans cette région: $1953-55,1973-77$ et 1980-86. Le paysage forestier a également été fortement influencé par les coupes forestières qui remontent au milieu du $19^{\mathrm{e}}$ siècle. L'étude se 
concentre sur des coupes datant de la fin des années '50. Les coupes non mécanisées en usage à ce moment assuraient une certaine protection de la régénération préétablie, de sorte que l'on peut poser l'hypothèse que ces coupes se comparent aux coupes avec protection de la régénération et des sols (CPRS) et aux coupes avec protection de la haute régénération (CPHR). La disponibilité de photographies aériennes couvrant le territoire a aussi été un autre critère pour retenir cette période.

\section{Classification écologique}

Le type écologique constitue le niveau hiérarchique du système de classification écologique du Québec le plus approprié à l'étude de la dynamique après coupe. Le type écologique constitue une unité relativement homogène en regard des variables du milieu physique, de la dynamique forestière et de la productivité. La codification pour les types écologiques est généralement constituée de deux lettres suivies de deux chiffres. Les deux premières lettres du code indiquent respectivement le type de couvert forestier et l'essence arborescente dominante de la végétation potentielle. Le premier chiffre sert également à caractériser la végétation potentielle tandis que le dernier chiffre exprime les caractéristiques physiques du milieu. Un chiffre additionnel peut être ajouté au besoin pour décrire des caractéristiques particulières du site (Gosselin et al. 2001).

La détermination des différents types écologiques associés aux peuplements étudiés a été effectuée en suivant les étapes décrites par Gosselin et al. (2001). Premièrement, les divers groupes écologiques d'espèces indicatrices ont été identifiés.
Par la suite, ces groupes écologiques d'espèces indicatrices ont été reliés aux espèces arborescentes afin de déterminer les végétations potentielles. Finalement, les différents types écologiques ont été déterminés en établissant des liens entre les groupes écologiques d'espèces indicatrices, les végétations potentielles et les types de milieux physiques.

Inventaire des peuplements issus de coupes de 1957 à 1963 Les secteurs considérés pour l'étude tirent leur origine de coupes de 1957 à 1963 et ont été localisés à l'aide des archives de la compagnie Abitibi-Consolidated Inc. L'année exacte de chacune des coupes a été déterminée à l'aide des reprises de croissance synchrones après la coupe, des cicatrices laissées par la coupe et des données historiques. À partir des photographies aériennes de 1957, les compositions des peuplements avant coupe ont pu être déterminées. Ces compositions ont été regroupées selon quatre principaux types de couvert : couvert d'épinette (EE), de sapin (SS), mélangé à dominance résineuse $(\mathrm{MR})$ et mélangé à dominance feuillue (MF).

Un échantillonnage stratifié en fonction du couvert avant coupe a été réalisé, à raison de 12 placettes par type de couvert. Le tableau 1 présente le nombre de relevés associés à chaque végétation potentielle et type écologique en fonction du type de couvert avant coupe. Les placettes établies ont une superficie de $400 \mathrm{~m}^{2}$. Les arbres marchands $(9,1 \mathrm{~cm}$ et plus) ont été dénombrés par classe de $2 \mathrm{~cm}$ sur l'ensemble de la superficie alors que le dénombrement des tiges non marchandes s'est fait sur une surface circulaire de $100 \mathrm{~m}^{2}$ au centre de la parcelle principale. De plus, au moins 12 tiges par

Tableau 1. Nombre de placettes selon la classification écologique et le type de couvert avant coupe

\begin{tabular}{|c|c|c|c|c|c|c|}
\hline \multirow[b]{2}{*}{ Végétation potentielle } & \multirow[b]{2}{*}{$\begin{array}{c}\text { Type } \\
\text { écologique }\end{array}$} & \multicolumn{5}{|c|}{ Type de couvert en 1957} \\
\hline & & $\begin{array}{l}\text { Mélangé à } \\
\text { dominance } \\
\text { feuillue }\end{array}$ & $\begin{array}{l}\text { Mélangé à } \\
\text { dominance } \\
\text { résineuse }\end{array}$ & Épinette & Sapin & Total \\
\hline \multirow[t]{2}{*}{ Sapinière à bouleau blanc } & MS21 & 3 & 1 & - & 2 & 22 \\
\hline & MS22 & 4 & 6 & - & 6 & \\
\hline \multirow[t]{2}{*}{ Sapinière à épinette noire } & RS22 & 3 & 3 & - & 1 & 14 \\
\hline & RS25 & 1 & 1 & 4 & 1 & \\
\hline \multirow[t]{2}{*}{ Pessière noire à mousses ou à éricacées } & RE21 & - & - & 7 & 1 & 9 \\
\hline & RE22 & - & - & 1 & - & \\
\hline \multirow[t]{3}{*}{ Bétulaie jaune à sapin } & MJ21 & 1 & - & - & - & 3 \\
\hline & MJ22 & - & 1 & - & 1 & \\
\hline & Total & 12 & 12 & 12 & 12 & 48 \\
\hline
\end{tabular}

MJ21 : Bétulaie jaune à sapin xérique-mésique de texture grossière

MJ22: Bétulaie jaune à sapin mésique de texture moyenne

MS21 : Sapinière à bouleau blanc xérique-mésique de texture grossière

MS22 : Sapinière à bouleau blanc mésique de texture moyenne

RE21: Pessière noire à mousses et éricacées xérique-mésique de texture grossière

RE22: Pessière noire à mousses ou à éricacées mésique de texture moyenne

RS22: Sapinière à épinette noire mésique de texture moyenne

RS25: Sapinière à épinette noire subhydrique de texture moyenne 
placette ont été choisies à titre d'arbres-études. Le choix des tiges s'est fait de façon a assurer la représentativité des espèces et dimensions de tiges présentes. Des rondelles ont été prélevées à $0,15 \mathrm{~m}, 1,3 \mathrm{~m}$ et $3 \mathrm{~m}$ pour tous les arbres-études. La hauteur totale a également été mesurée.

Dans chacune des 48 placettes retenues, 25 sous-parcelles de $4 \mathrm{~m}^{2}$ ont été implantées dans le but de déterminer le coefficient de distribution à différents moments et de calculer un indice de diamètre 10 ans après la coupe. Pour ce faire, des rondelles à la souche ont été prélevées pour chacune des tiges dominantes, vivantes et mortes, dans chacune des sousparcelles et ce, pour chaque essence présente. Il s'agissait alors d'inventorier les tiges dominantes vivantes, et si la dimension d'une tige morte de la sous-parcelle laissait croire qu'elle avait pu dominer ou occuper une période de temps non couverte par la tige dominante actuelle, cette tige morte était également inventoriée.

Les rondelles prélevées ont été séchées et sablées, puis elles ont été analysées à l'aide du système automatique de mesure WinDendro'. Les largeurs de cernes annuels ont été mesurées sur quatre rayons disposés à 90 degrés les uns des autres. Les changements marqués de la largeur ou de la teinte des cernes annuels ont été notés. Ces observations ont permis de déterminer par interdatation la période d'établissement des individus morts et des tiges fortement affectées par la tordeuse, présentant des cernes manquants (Morin 1994). Enfin, un inventaire des plantes de sous-bois et des conditions de station a été réalisé dans chacune des placettes pour procéder à leur classification écologique.

\section{Analyses statistiques}

Dans un premier temps, nous avons voulu déterminer s'il existait un lien entre le couvert d'origine, et l'abondance et la structure de la régénération, dix ans après la coupe. Pour ce faire, des analyses de variance (Proc GLM, SAS Institute Inc. 1989) ont été réalisées pour chaque essence et groupe d'essences. Lorsque des différences significatives étaient notées, le test de comparaisons multiples de Duncan a été utilisé pour déterminer entre quels types de couvert des différences étaient observables. Une transformation « racine carrée " a été appliquée lorsque les variances étaient hétérogènes. Les données estimées à dix ans ont été retenues puisqu'elles correspondent au second suivi recommandé dans le Manuel d'Aménagement Forestier (MRNFP 2003) pour la production de résineux en forêt mélangée. Compte tenu du fort dynamisme de la végétation dans les premières années après la coupe et de la difficulté à bien retracer les petites tiges mortes peu de temps après la coupe, le potentiel de prédiction du rendement a été jugé meilleur à ce moment que plus tôt après coupe.

Pour chaque espèce ou groupe d'espèce, deux variables ont été utilisées pour caractériser la régénération, soit le coefficient de distribution et un indice de diamètre. Le coefficient de distribution représente le pourcentage des sousparcelles où l'espèce est présente. C'est la variable diagnostique la plus couramment utilisée pour juger du niveau de régénération. Quant à l'indice de diamètre (équation 1), il représente le diamètre quadratique des 1000 plus grosses tiges à l'hectare (Riopel 1999). Cet indice a été calculé dix ans après coupe afin d'établir le lien entre la taille et la structure de la régénération et le rendement 38 ans après coupe.

\section{[1] $\quad D_{10}=\sqrt{\frac{\sum n_{i} d_{i}^{2}}{1000}}$}

où $\mathrm{ID}_{10}$ est l'indice de diamètre dix ans après coupe, $\mathrm{n}_{\mathrm{i}}$ est le nombre de tiges de la classe ${ }_{i}$ et $\mathrm{d}_{\mathrm{i}}$ est la classe ${ }_{i}$ du diamètre à hauteur de poitrine. L'intérêt de cet indice est qu'il combine abondance et structure de la régénération dans une même variable. Il s'est avéré un outil efficace pour expliquer la production des peuplements à partir des caractéristiques de la régénération (Riopel 1999).

Les échantillons prélevés à hauteur de souche dans les sous-parcelles de $4 \mathrm{~m}^{2}$ ont été utilisés pour caractériser les peuplements à 10 ans. Afin d'obtenir le diamètre à hauteur de poitrine (DHP) de ces tiges à dix ans, une régression entre ce dernier et le diamètre à hauteur de souche (DHP-DHS) dix ans après coupe a été construite à partir des données provenant des arbres-études. De cette façon, il a été possible d'obtenir le DHP des tiges dominant les sous- parcelles de 4 $\mathrm{m}^{2}$ et seules les dix plus grosses de la placette, de chaque essence ou regroupement d'essences, ont servi au calcul de l'indice.

L'évolution du coefficient de distribution a été analysée comme une série de mesures répétées selon la procédure décrite par Meredith et Stehman (1991). Ainsi, les coefficients de régression décrivant les effets linéaires, quadratiques et cubiques du temps ont été calculés pour les données provenant d'une parcelle donnée. Par la suite, ces coefficients ont été soumis à une analyse de variance en incorporant le type écologique comme variable explicative. Les types écologiques ont été comparés deux à deux en appliquant la correction de Bonferroni (Berny 1999).

Un test de chi-carré a été utilisé pour vérifier le lien entre le type de peuplement d'origine et le type de peuplement actuel. Le chi-carré de chaque cellule a permis de vérifier les types de peuplements touchés lorsqu'une différence était observée. Deux analyses distinctes ont été effectuées. La première a permis d'examiner l'évolution des composantes feuillue et résineuse dans les différents peuplements tandis que, pour la seconde analyse, l'emphase a été portée sur l'évolution du sapin par rapport à l'épinette.

La régression linéaire simple a été utilisée pour expliquer le volume total 38 ans après coupe en fonction du coefficient de distribution et de l'indice de diamètre dix ans après la coupe. Les volumes totaux des tiges marchandes et non marchandes ont été utilisés en raison du stade de prématurité des peuplements inventoriés qui implique qu'un nombre important de tiges n'a pas atteint un diamètre marchand.

En vue d'estimer le volume de chaque tige, un modèle non-linéaire de la hauteur totale en fonction du diamètre à hauteur de poitrine (DHP) a été calibré pour chaque essence en utilisant le modèle de Bégin et Raulier (1995). Puisque l'âge actuel des peuplements varie de 38 à 43 ans, une correction a été apportée pour estimer les diamètres à 38 ans. Ainsi, le diamètre des tiges ayant subi une coupe il y a plus de 38 ans a été diminué d'une valeur correspondant à la moyenne des accroissements après les 38 premières années pour une espèce et une placette donnée. La production ligneuse a ensuite été estimée pour chaque peuplement en calculant le volume sans écorce à l'aide d'un tarif de cubage à deux entrées (Perron 1985). 


\section{Résultats \\ Lien entre le couvert d'origine et certaines caractéristiques de régénération à dix ans}

Les résultats des analyses de variance démontrent que le peuplement d'origine a un effet significatif sur les coefficients de distribution à dix ans, et ce pour le groupement d'essences feuillues et pour toutes les essences prises individuellement $(\operatorname{Pr}<0,05)$. Seul le coefficient de distribution de l'ensemble des essences résineuses ne présente pas de différences entre les types de couvert $(\operatorname{Pr}=0,18)$.

Les tests de comparaisons multiples révèlent que le coefficient de distribution de la régénération en épinette noire (Picea mariana (Mill.) BSP) à dix ans est supérieur pour les pessières noires. Pour le sapin baumier, le test de Duncan ne permet pas de détecter de différences entre les sapinières, les peuplements mélangés à dominance résineuse et les peuplements mélangés à dominance feuillue. Les résultats permettent toutefois d'affirmer que la régénération en sapin baumier est mieux distribuée dans les peuplements mélangés que dans les pessières noires, le maximum étant atteint pour les peuplements mélangés à dominance feuillue avec un coefficient de distribution de $52 \%$.

Dans le cas des essences feuillues, on constate que le coefficient de distribution 10 ans après la coupe est beaucoup plus faible dans les pessières noires. De plus, des différences sont observables entre les sapinières et les peuplements mélangés à dominance feuillue, ces derniers étant caractérisés par un coefficient de distribution de la régénération à dix ans plus élevé de $23 \%$. Il n'existe pas de différence entre les deux types de peuplements mélangés. Le coefficient de distribution du bouleau blanc (Betula papyrifera Marsh.) est inférieur dans les pessières par rapport aux autres types de peuplements. Quant au peuplier faux-tremble (Populus tremuloides Michx.), il est moins abondant dans les peuplements résineux que dans les peuplements mélangés à dominance feuillue.

Le même type d'analyse a été réalisé pour comparer les indices de diamètre obtenus à dix ans en fonction du couvert d'origine. Les résultats démontrent que les indices de diamètre pour les résineux et pour les principales essences résineuses analysées individuellement ne different pas entre eux $(\operatorname{Pr}>0,05)$. À l'inverse, les résultats des analyses de variance pour les feuillus et les principales essences feuillues présentent des différences significatives $(\operatorname{Pr}<0,05)$. L'indice de diamètre feuillu à dix ans est significativement plus faible dans les pessières. Pour les essences feuillues prises individuellement, les patrons sont moins nets mais on n'observe aucune différence entre les sapinières et les pessières.

\section{Évolution des coefficients de distribution des tiges dominantes depuis la coupe}

L'analyse de l'évolution des coefficients de distribution en fonction du type de couvert avant coupe et du type écologique a permis de constater que de façon générale, il y a augmentation des coefficients de distribution sur une période d'environ 10 ans suivant la coupe (Fig. 1). Il y a ensuite stabilisation des coefficients dans le temps pour enfin observer une diminution d'importance variable selon les types écologiques, environ 25 ans après la coupe pour les coefficients de distribution résineux. L'évolution des coefficients de distribution résineux associés aux types écologiques résineux et mélangés se distingue par une baisse plus prononcée chez ces derniers. Dans le cas des feuillus, seul le type écologique de la sapinière à bouleau blanc xériquemésique de texture grossière (MS21) ne présente pas d'augmentation aussi marquée dans les premiers 10 ans suivant la coupe. L'analyse des écarts types (Fig. 1) permet d'affirmer que les peuplements composés d'une forte proportion d'épinette noire présentent la plus faible variabilité, et ce, malgré des écarts importants en début de révolution. On remarque également que pour les coefficients de distribution résineux, les types écologiques résineux (RE21, RS22 et RS25) présentent une variabilité plus faible que les types écologiques mélangés (MS21, MS22).

\section{Analyse de l'évolution du coefficient de distribution en fonction du type écologique}

Les principaux résultats de l'analyse en mesures répétées effectuée sur les tiges dominantes permettant de décrire les courbes d'évolution des coefficients de distribution en fonction des types écologiques sont présentés au tableau 2. On constate que seul le type écologique a un effet significatif sur le coefficient de distribution associé au groupement d'essences résineuses et aux essences qui le composent. Cela suggère que les coefficients de distribution résineux présentent des différences initiales et que celles-ci se maintiennent dans le temps. Dans le cas des feuillus, on observe que l'interaction entre le type écologique et le temps influence significativement le coefficient de distribution. Ces interactions démontrent donc que les coefficients de distribution feuillus évoluent différemment entre les types écologiques en fonction du temps.

La représentation graphique de l'évolution des coefficients de distribution facilite la visualisation des différences significatives entre les types écologiques (Fig. 2). Les résultats démontrent que, pour l'épinette noire, les types écologiques résineux inventoriés (RE21, RS22 et RS25) se distinguent des types écologiques mélangés (MS21 et MS22) par des coefficients de distribution plus élevés tout au long de l'horizon ( $\mathrm{Pr}<0,05)$. Pour le sapin baumier, on constate que les types écologiques appartenant à la végétation potentielle de la sapinière à épinette noire (RS2) présentent des courbes d'évolution supérieures à la végétation potentielle de la pessière noire (RE2) ( $\operatorname{Pr}<0,05)$. Aucune différence significative n'a été observée entre les niveaux des courbes d'évolution du sapin baumier sur les types écologiques de la sapinière à épinette noire (RS2) et ceux de la sapinière à bouleau blanc (MS2) ( $\operatorname{Pr}>0,05)$. Enfin, lorsque l'on considère les deux résineux ensemble, seul le type écologique de la sapinière à bouleau blanc mésique de texture moyenne (MS22) se distingue des types écologiques de la sapinière à épinette noire (RS22 et RS25) par des coefficients de distribution plus faibles d'environ 20\% sur tout l'horizon ( $\mathrm{Pr}$ $=0,0246$ et $\operatorname{Pr}=0,0436$ ).

Dans le cas des feuillus, les résultats démontrent que la sapinière à bouleau blanc mésique de texture moyenne (MS22) differe de la pessière noire à mousses et éricacées xérique-mésique de texture grossière (RE21), et ce, pour les trois types d'effet $(\mathrm{Pr}<0,05)$. On remarque également que linéairement, la sapinière à épinette noire mésique de texture moyenne (RS22) se différencie des autres types écologiques à 


\section{Pessières noires}
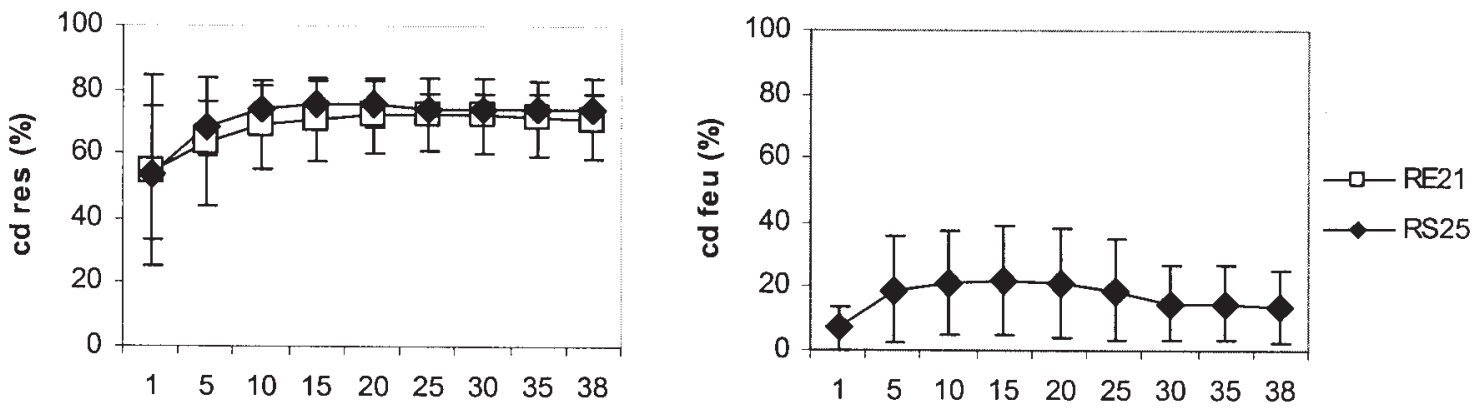

Mélangé à dominance feuillue
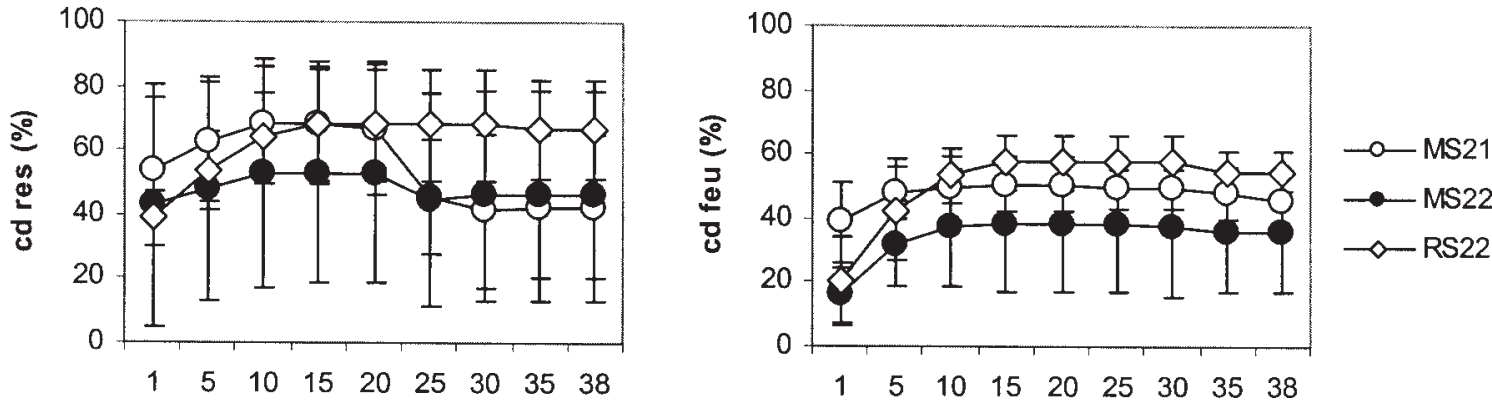

Mélangés à dominance résineuse
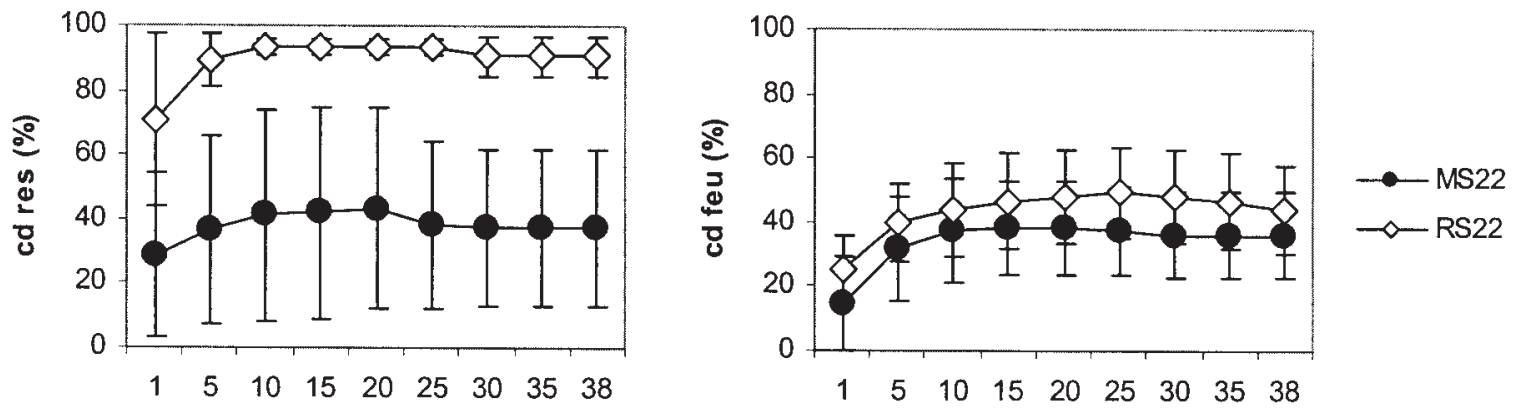

Sapinières
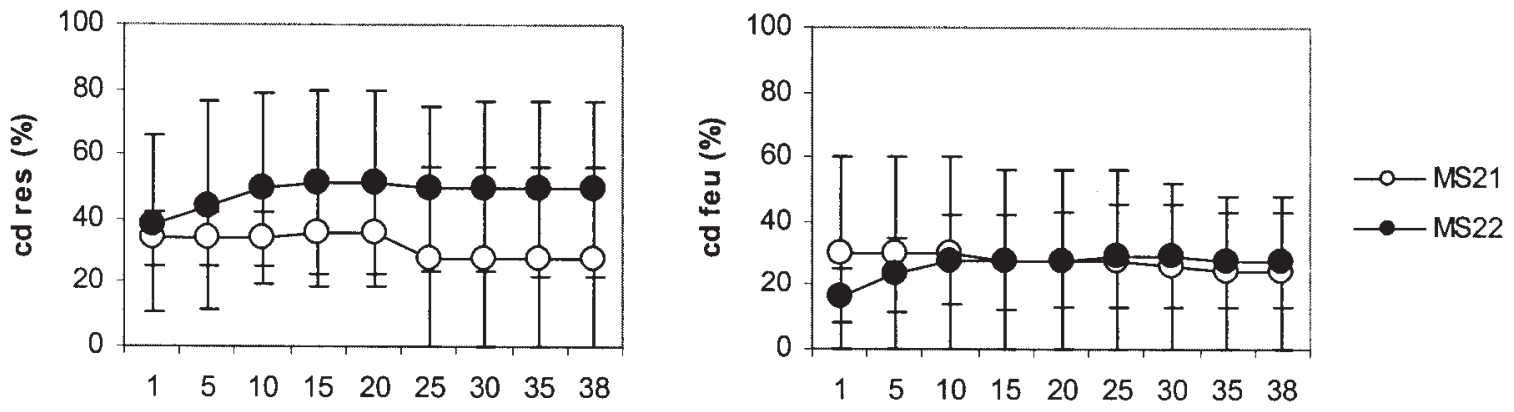

Fig. 1. Évolution des coefficients de distribution résineux (cdres) et feuillu (cdfeu) en fonction du type de couvert avant coupe et des types écologiques. 
Tableau 2. Analyse statistique de l'évolution temporelle des coefficients de distribution des tiges dominantes en fonction du type écologique (RES : résineux; SAB : sapin; EPN : épinette noire; FEU : feuillus; BOP : bouleau blanc; PET : peuplier faux-tremble).

\begin{tabular}{llcc}
$\begin{array}{l}\text { Essence ou } \\
\text { groupe d'essences }\end{array}$ & Type d'effet & $F$ & Pr \\
\hline RES & Type écologique & 5,08 & 0,0022 \\
SAB & Type écologique & 5,03 & 0,0023 \\
& EPN & 19,73 & $<0,0001$ \\
& Type écologique & & 0,0002 \\
FEU & Type écologique*temps (linéaire) & 7,22 & 0,0072 \\
& Type écologique*temps (quadratique) & 4,10 & 0,0245 \\
& Type écologique*temps (cubique) & 3,15 & 0,0041 \\
BOP & Type écologique*temps (linéaire) & 4,55 & 0,0013 \\
& & & 0,0302 \\
PET & Type écologique & 5,51 & 0,0107 \\
& Type écologique*temps (linéaire) & 2,99 & 3,79 \\
\end{tabular}

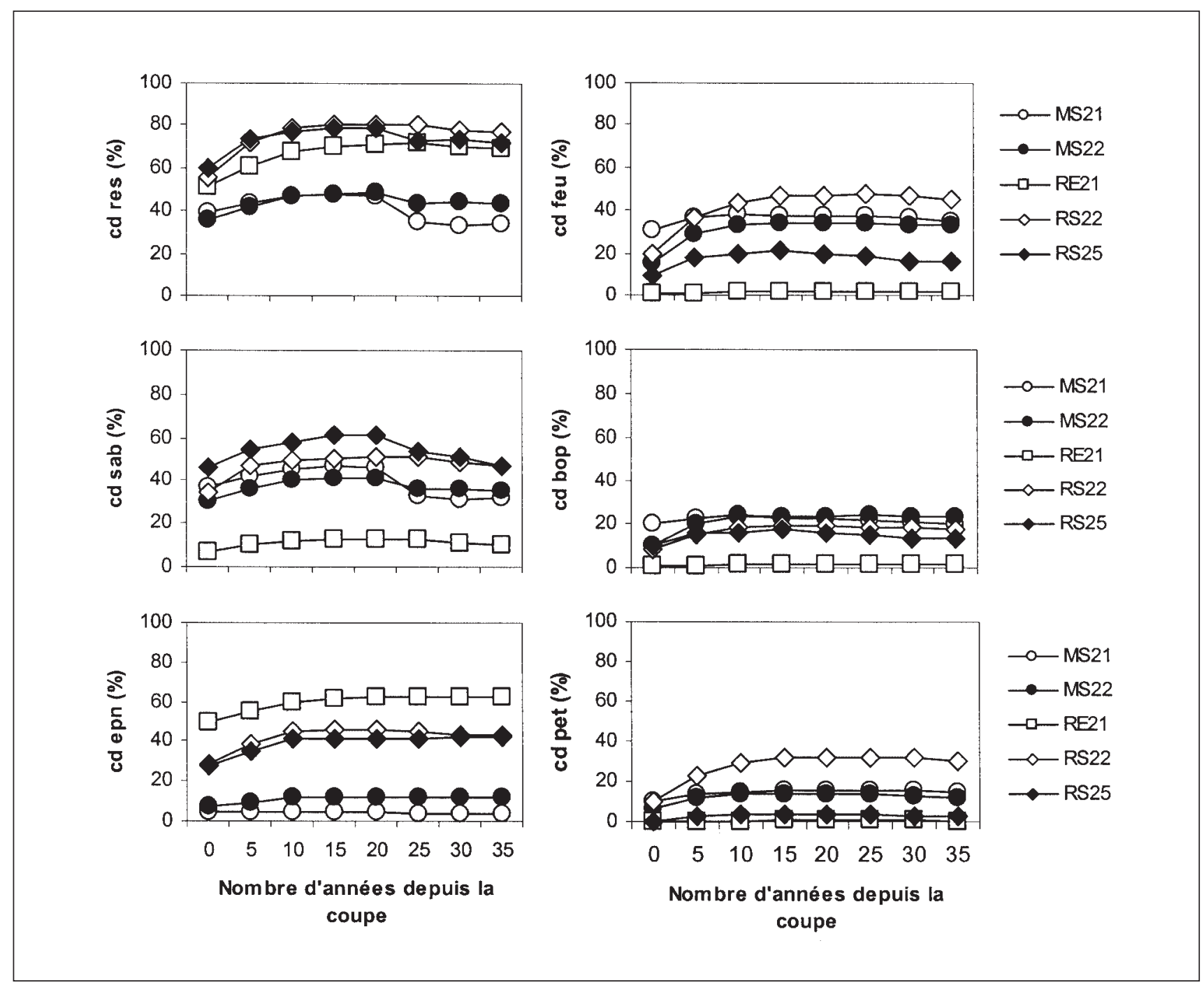

Fig. 2. Évolution des coefficients de distribution par essence et groupe d'essences (res : résineux; sab : sapin; epn : épinette noire; feu : feuillus; bop : bouleau blanc; pet : peuplier faux-tremble) en fonction des types écologiques. 
Tableau 3. Tableau du test de chi-carré par cellule individuelle pour la tendance au niveau de la composition en fonction du type de couvert avant coupe (EE : pessières à épinette noire; MF : mélangé à dominance feuillue; MR : mélangé à dominance résineuse, SS : sapinières].

\begin{tabular}{|c|c|c|c|c|c|}
\hline & \multicolumn{3}{|c|}{ Tendance au niveau de la composition } & \multirow[b]{2}{*}{ Total } & \multirow{24}{*}{$\begin{array}{l}\text { Contenu des cellules: } \\
\text { Fréquence; } \\
\text { Valeur du chi-carré par cellule } \\
(1 \mathrm{dl}, 0.95=3,841) ; \\
\text { Pourcentage; } \\
\text { Pourcentage par type de couvert } \\
\text { Pourcentage par tendance. }\end{array}$} \\
\hline & Feuillue & Enrésinement & Stable & & \\
\hline \multirow{5}{*}{$\mathrm{EE}$} & 0 & 0 & 12,00 & 12 & \\
\hline & 4,5 & 1,75 & 6,7935 & - & \\
\hline & 0 & 0 & 25,00 & 25,00 & \\
\hline & 0 & 0 & 100,00 & - & \\
\hline & 0 & 0 & 52,17 & - & \\
\hline \multirow{5}{*}{ MF } & 2 & 4 & 6 & 12 & \\
\hline & 1,3889 & 2,8929 & 0,0109 & - & \\
\hline & 4,17 & 8,33 & 12,50 & 25,00 & \\
\hline & 16,67 & 33,33 & 50,00 & - & \\
\hline & 11,11 & 57,14 & 26,09 & - & \\
\hline \multirow{5}{*}{ MR } & 7 & 3 & 2 & 12 & \\
\hline & 1,3889 & 0,8929 & 2,4457 & - & \\
\hline & 14,58 & 6,25 & 4,17 & 25,00 & \\
\hline & 58,33 & 25,00 & 16,67 & - & \\
\hline & 38,89 & 42,86 & 8,70 & - & \\
\hline \multirow{5}{*}{ SS } & 9 & 0 & 3 & 12 & \\
\hline & 4,5 & 1,75 & 1,3152 & - & \\
\hline & 18,75 & 0,00 & 6,25 & 25,00 & \\
\hline & 75,00 & 0,00 & 25,00 & - & \\
\hline & 50,00 & 0,00 & 13,04 & - & \\
\hline \multirow[t]{2}{*}{ Total } & 18 & 7 & 23 & 48 & \\
\hline & 37,50 & 14,58 & 47,92 & 100,00 & \\
\hline
\end{tabular}

Nota : Les valeurs significatives par type de couvert au niveau de signification de $5 \%$ apparaissent en gras.

l'exception de la sapinière à bouleau blanc mésique de texture moyenne (MS22) ( $\operatorname{Pr}<0,05)$. En plus de l'effet linéaire, on observe que les coefficients de distribution pour les feuillus de la sapinière à épinette noire mésique de texture moyenne (RS22) sont significativement différents de ceux de la pessière noire à mousses et éricacées xérique-mésique de texture grossière (RE21) selon l'effet quadratique du temps ( $\mathrm{Pr}=$ $0,0065)$. On remarque une moins grande installation du bouleau blanc dans la pessière noire à mousses et éricacées xérique-mésique (RE21) que dans la sapinière à bouleau blanc mésique de texture moyenne (MS22). On observe aussi une plus forte installation du peuplier faux-tremble au cours des dix premières années dans la sapinière à épinette noire mésique de texture moyenne (RS22).

Effet du couvert avant coupe sur la composition de la strate de retour

L'analyse du chi-carré a permis de déceler certaines tendances quant aux changements de composition associés aux peuplements d'origine de la pessière et de la sapinière. En effet, le tableau 3 démontre que les sapinières subissent une augmentation des feuillus $\left(\chi^{2}=4,5\right)$ tandis que les pessières demeurent stables $\left(\chi^{2}=6,7935\right)$ en termes de composition. Les valeurs non significatives au niveau des cellules individuelles démontrent que les peuplements mélangés se comportent comme la moyenne. On observe ainsi que 58\% des peuplements mixtes à dominance résineuse ont vu leur composante feuillue augmenter.

Afin de tester l'importance du phénomène d'ensapinage à la suite de la coupe, une analyse similaire a été réalisée en séparant les deux principales essences résineuses. À première vue, le sapin baumier semble occuper une place plus importante dans les peuplements de retour. Parmi les placettes dans lesquelles on observe un changement dans la proportion sapin baumier/épinette, on note que dans $73 \%$ des cas (19 parcelles sur 26), le sapin occupe une place plus importante comparativement au peuplement d'origine. Malgré ce constat, l'analyse du chi-carré n'a détecté aucune différence significative, ce qui signifie que cette augmentation ne serait pas liée au couvert d'origine. Â noter que les placettes dont la composante résineuse avait disparu en 2000 n'ont pas été considérées dans l'analyse, puisqu'elles n'informent pas sur l'augmentation de la proportion de sapin.

Estimation du rendement en fonction du coefficient de distribution et de l'indice de diamètre à dix ans

Les volumes marchands moyens mesurés en 2000 varient de 37 $\mathrm{m}^{3} /$ ha pour les peuplements mélangés à dominance feuillue avant coupe, jusqu'à $65 \mathrm{~m}^{3} /$ ha pour ceux originant de la coupe de sapinières. Les volumes marchands feuillus sont négligeables dans les pessières alors qu'ils se situent entre 43 et $59 \mathrm{~m}^{3} / \mathrm{ha}$ dans les autres types de peuplements. Puisque de nombreuses 

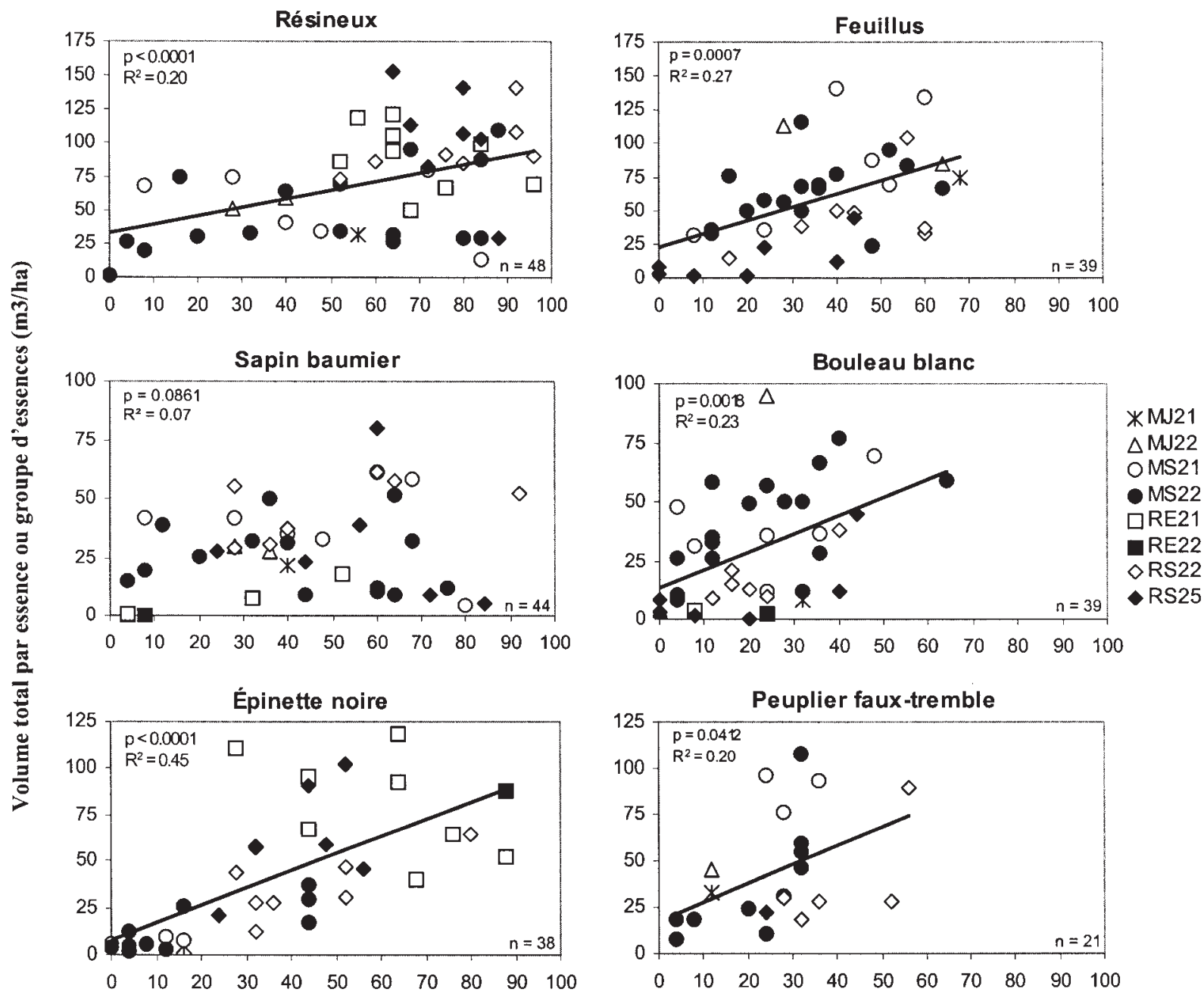

Coefficient de distribution de la régénération par essence ou groupe d'essences (\%)

Fig. 3. Régressions linéaires entre le coefficient de distribution à dix ans et le volume total à 38 ans.

tiges n'ont pas encore atteint le diamètre marchand, l'analyse du rendement se concentre sur le volume total.

Les figures 3 et 4 présentent le volume total à 38 ans en fonction du coefficient de distribution et de l'indice de diamètre à 10 ans. Dans tous les cas à l'exception du sapin baumier ( $\left.\mathrm{Pr}=0,086 ; \mathrm{R}^{2}=0,07\right)$, on observe que le coefficient de distribution à 10 ans influence significativement le rendement futur des peuplements, et ce, pour tous les types écologiques confondus (Fig. 3). Par contre, la faiblesse des coefficients de détermination obtenus indique qu'une variabilité importante existe. Pour l'analyse portant sur le groupement des essences résineuses, les types écologiques résineux (RE21, RS22, RS25) ont généralement de bons coefficients de distribution à dix ans et une forte proportion de ces placettes se situe au-dessus de la courbe de tendance.

L'importance du coefficient de distribution de l'épinette noire en bas âge sur le rendement futur du peuplement est démontrée par l'obtention d'un résultat significatif pour tous les types écologiques confondus $\left(\operatorname{Pr}<0,0001 ; R^{2}=0,45\right)$. De façon générale, les rendements supérieurs sont observés pour les placettes appartenant aux types écologiques de la sapinière à épinette noire subhydrique de texture moyenne (RS25) et de la pessière noire à mousses et à éricacées xérique-mésique de texture grossière (RE21). Les placettes appartenant au type de milieu mésique de texture moyenne (MS22 et RS22) se retrouvent en forte proportion sous la courbe de tendance.

L'analyse de l'ensemble des placettes sans égard aux types écologiques révèle que le coefficient de distribution des feuillus à dix ans influence le volume total en feuillus dans les peuplements 38 ans après la coupe $\left(\mathrm{Pr}=0,0007 ; \mathrm{R}^{2}=0,27\right)$. Dans le cas présent, on remarque que les placettes appartenant à la végétation potentielle de la sapinière à bouleau blanc (MS2) sont généralement situées au-dessus de la courbe de tendance alors que celles appartenant à la 

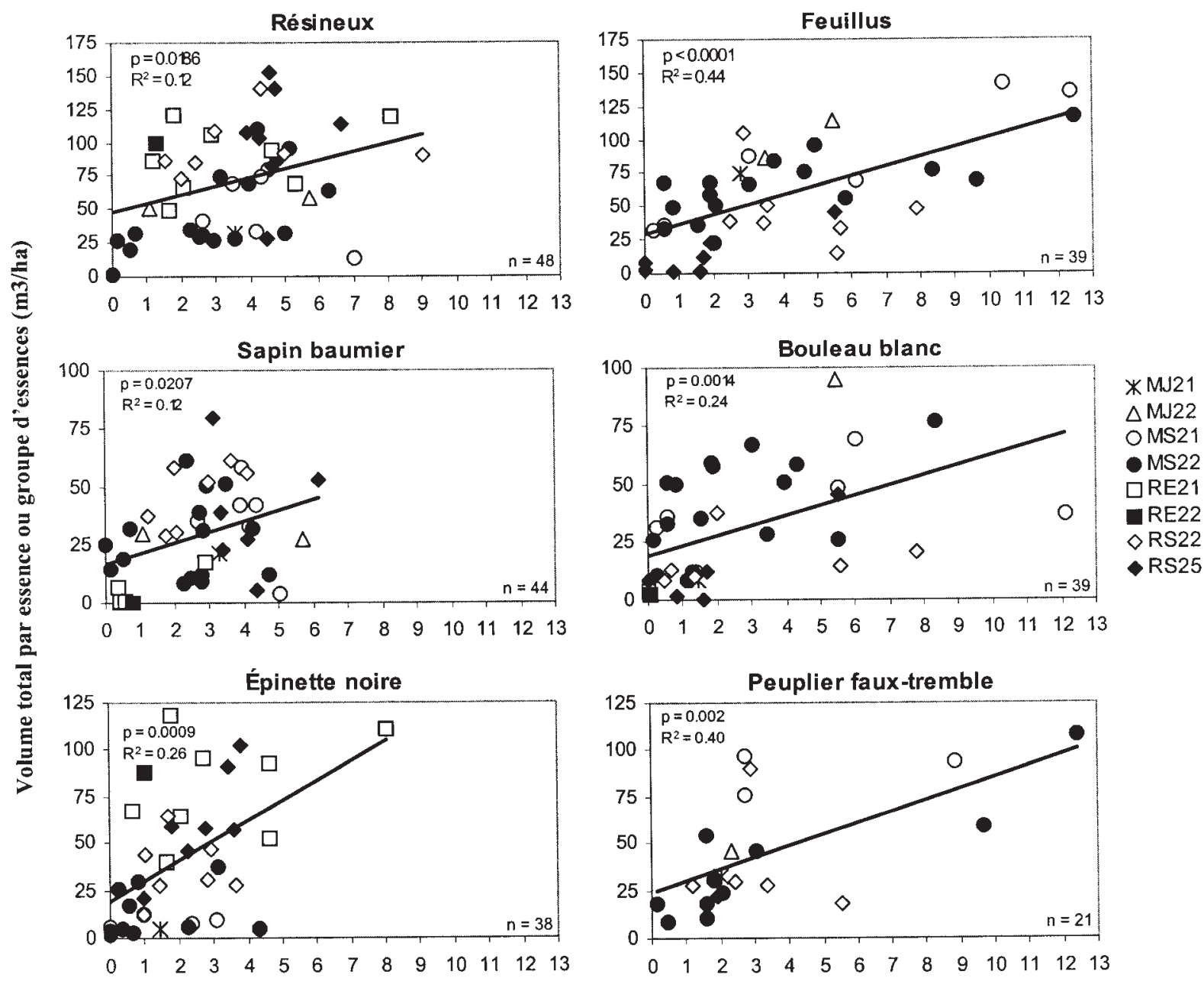

Indice de diamètre par essence ou groupe d'essences $(\mathrm{cm})$

Fig. 4. Régressions linéaires entre l'indice de diamètre à dix ans et le volume total à 38 ans.

sapinière à épinette noire (RS2) se retrouvent en très forte proportion sous la courbe et ce, pour des coefficients de distribution comparables.

Les analyses effectuées en considérant chacun des types écologiques révèlent que le coefficient de distribution à 10 ans est efficace pour prédire le rendement particulièrement dans le cas de la sapinière à bouleau blanc mésique de texture moyenne (MS22). En effet, des relations significatives ont été observées pour les résineux $\left(\mathrm{Pr}=0,05 ; \mathrm{R}^{2}=0,24\right)$, l'épinette noire $\left(\operatorname{Pr}=0,0002 ; \mathrm{R}^{2}=0,68\right)$, le bouleau blanc $(\operatorname{Pr}=0,02$; $\left.\mathrm{R}^{2}=0,33\right)$ et le peuplier faux-tremble $\left(\operatorname{Pr}=0,03 ; \mathrm{R}^{2}=0,47\right)$. À noter que pour l'épinette noire, une relation a également été décelée dans le cas de la sapinière à épinette noire mésique de texture moyenne ( $R S 22)\left(\operatorname{Pr}=0,02 ; R^{2}=0,88\right)$. La taille de l'échantillon demeure toutefois faible à ce niveau d'analyse.
Tout comme le coefficient de distribution pour l'ensemble des résineux, l'indice de diamètre à dix ans influence le rendement obtenu à 38 ans $(\operatorname{Pr}=0,0186)$ même si la variabilité demeure importante $\left(\mathrm{R}^{2}=0,12\right)$ (Fig. 4). Encore une fois, malgré des indices de diamètre similaires, on observe que les types écologiques résineux (RE21, RS22, RS25) présentent généralement de meilleurs rendements. Pour le sapin baumier, les résultats démontrent une relation significative entre l'indice de diamètre et le rendement $(\mathrm{Pr}=$ 0,$\left.021 ; \mathrm{R}^{2}=0,12\right)$, alors que pour le coefficient de distribution, aucune relation n'avait été décelée. À partir du graphique, on remarque que les placettes de la sapinière à épinette noire mésique de texture moyenne (RS22) sont toujours situées au-dessus de la courbe de tendance alors que celles de la pessières noires à mousses et à éricacées (RE21) se retrouvent au-dessous. Le rendement de l'épinette noire à 38 
ans est également influencé par l'indice de diamètre à dix ans de cette essence pour l'analyse globale de tous les types écologiques $\left(\mathrm{Pr}=0,0009 ; \mathrm{R}^{2}=0,26\right)$.

L'indice de diamètre des feuillus a aussi un effet significatif sur le rendement en volume total à 38 ans $\left(\operatorname{Pr}<0,0001 ; \mathrm{R}^{2}\right.$ $=0,44)$. Tout comme pour le coefficient de distribution, les placettes appartenant à la végétation potentielle de la sapinière à bouleau blanc (MS2) se retrouvent généralement au-dessus de la courbe de tendance alors que celles de la sapinière à épinette noire (RS2) se situent majoritairement en dessous. L'indice de diamètre est aussi en lien avec le rendement pour le bouleau blanc $(\operatorname{Pr}=0,0014)$ et le peuplier faux-tremble $(\operatorname{Pr}=0,002)$.

En considérant chacun des types écologiques séparément, on observe certaines tendances quant à la capacité de l'indice de diamètre à prédire le rendement en volume à 38 ans. Des relations significatives ont en effet été notées dans le cas de la sapinière à bouleau blanc mésique de texture moyenne (MS22) pour les résineux ( $\left.\mathrm{Pr}=0,003 ; \mathrm{R}^{2}=0,47\right)$, les feuillus $\left(\operatorname{Pr}=0,003 ; \mathrm{R}^{2}=0,49\right)$, le bouleau blanc $\left(\operatorname{Pr}=0,04 ; \mathrm{R}^{2}=\right.$ $0,26)$ et le peuplier faux-tremble $\left(\operatorname{Pr}=0,0007 ; \mathrm{R}^{2}=0,78\right)$. Des liens significatifs existent pour l'épinette noire $(\mathrm{Pr}=0,02$ ; $\left.\mathrm{R}^{2}=0,68\right)$, les feuillus $\left(\operatorname{Pr}=0,005 ; \mathrm{R}^{2}=0,82\right)$ et le bouleau blanc $\left(\mathrm{Pr}=0,01 ; \mathrm{R}^{2}=0,83\right)$ dans la sapinière à épinette noire subhydrique de texture moyenne (RS25). Finalement, une relation a été observée pour les feuillus sur le type écologique de la sapinière à bouleau blanc xérique-mésique de texture grossière (MS21) $\left(\mathrm{Pr}=0,006 ; \mathrm{R}^{2}=0,87\right)$.

\section{Discussion}

Un des défis posés à l'aménagiste forestier consiste à prévoir la composition et le rendement des peuplements de seconde venue. Nous avons ainsi pu constater que, pour un même type de peuplement avant coupe, la composition et le rendement pouvaient être très variables. Nous avons observé que le type de peuplement de seconde venue différait selon le peuplement d'origine. Les rendements se sont avérés très variables et le coefficient de distribution, principal critère de décision en évaluation de la régénération, a démontré une efficacité souvent faible à prédire le rendement à 38 ans. Malgré certaines améliorations liées à l'utilisation d'un indice de diamètre afin de décrire la structure de la régénération, notre capacité à prédire le rendement est demeurée plutôt limitée.

La difficulté à prédire la composition et le rendement des peuplements de seconde venue découle de plusieurs facteurs. Il est généralement reconnu que la régénération préétablie en résineux joue un rôle important dans les strates de retour, particulièrement lorsque les méthodes de coupe permettent sa protection, comme c'était généralement le cas avant la mécanisation des coupes. L'abondance de la régénération préétablie est dépendante de la présence de semenciers pour la régénération sexuée ou encore d'arbres-mères pour la régénération végétative. En ce sens, elle est étroitement reliée à la nature du couvert avant coupe. La coupe qui s'ensuivra aura pour effet de réduire l'abondance de la régénération préétablie et cette baisse sera fonction des méthodes de coupe utilisées. Comme les méthodes de coupe sont les mêmes pour l'ensemble des sites étudiés, nous devrions nous attendre à observer un lien entre le couvert d'origine et la régénération présente immédiatement après coupe et éventuellement avec celle présente une dizaine d'années plus tard.

L'examen des coefficients de distribution des différentes essences à dix ans confirme l'existence d'un lien avec le couvert d'origine. La régénération en épinette noire est nettement plus abondante dans les pessières noires alors que le sapin y était moins abondant que dans les peuplements mélangés. Plusieurs auteurs ont constaté que la régénération préétablie en épinette noire était surtout constituée de marcottes, de sorte que la présence de l'épinette au sein du couvert influence fortement son abondance au sein de la régénération (Doucet 1989, Ruel 1989, Sarrasin 1991, Lussier et al. 1992, Morin et Gagnon 1992, Paquin et Doucet 1992a, Ruel 1992a). La meilleure capacité de dispersion du sapin baumier par rapport au marcottage ainsi que son agressivité à s'installer sous couvert (Hatcher 1960, Frank 1990) pourraient expliquer l'abondante régénération en sapin baumier autant dans les peuplements issus des couverts mélangés que dans ceux issus de sapinières. Même si une régénération complémentaire en épinette s'est installée, on constate que cette installation ne variait pas selon le type écologique comme en témoigne l'absence d'interaction avec l'effet du temps (tableau 2). La même observation vaut aussi pour la régénération en sapin et pour celle de l'ensemble des résineux. L'augmentation des coefficients de distribution résineux au cours des dix premières années suivant la coupe confirme les résultats obtenus par Symons (1996) et Ruel et al. (1998) qui sous-tendent que, malgré l'importance de la régénération résineuse préétablie, le recrutement d'une régénération additionnelle est également possible. Le même phénomène a été observé dans les pessières noires par Richardson (1975), Newton (1990), Pominville et Ruel (1995), Groot (1996) et Pothier (1996).

La situation est toutefois fort différente pour la régénération des feuillus. Les deux espèces les plus abondantes, le bouleau blanc et le peuplier faux tremble, sont deux espèces intolérantes à l'ombre. Leur régénération préétablie est ainsi généralement faible, sauf lorsque les peuplements ont subi une perturbation permettant une mise en lumière du parterre forestier (Ruel et Huot 1993). Ceci explique que les coefficients de distribution observés après coupe sont généralement faibles. Le cas de la sapinière à bouleau blanc xérique-mésique de texture grossière (MS21) fait cependant exception, puisque des coefficients en feuillus de l'ordre de $40 \%$ ont été observés immédiatement après coupe. Notons qu'un épisode épidémique de tordeuse des bourgeons a été rapporté pour 1953-1955 et que les sapinières sur milieux xériques ou à texture grossière sont reconnues comme plus vulnérables à la tordeuse des bourgeons de l'épinette (Archambault et al. 1990, Dupont et al. 1991), Par ailleurs, dans la présente étude, on assiste à une installation quelquefois importante des feuillus dans les dix premières années, ce qui rejoint les observations de plusieurs auteurs (Hatcher 1960, Richardson 1975, Boivin 1977, Hibbs 1983, White 1991, Pothier 1996). Dans le cas du bouleau, l'installation se ferait principalement par semis et serait favorisée par les grandes distances de dispersion de l'espèce. Pour le peuplier, le drageonnement jouerait un rôle déterminant (Frey et al. 2003). L'installation des feuillus, ainsi que celle des espèces prises individuellement, varie en 
fonction du type écologique. On constate ainsi que les conditions hydriques extrêmes, souvent favorables à l'établissement de l'épinette noire, ne permettent pas l'établissement et le développement optimal des feuillus intolérants (Perala 1990, Safford et al. 1990, Viereck et Johnston 1990). Ces conditions se retrouvent particulièrement sur les types écologiques de la sapinière à épinette noire subhydrique de texture moyenne (RS25) et de la pessière noire à mousses et éricacées xérique-mésique de texture grossière (RE21). Plusieurs études ont aussi démontré que, sur des sites similaires, lorsque la composante feuillue s'établit après la coupe, elle présente un déclin au cours des cinq à dix premières années (Sarrasin 1991, Pothier 1996, Ruel et al. 1998). À la suite de la coupe, les feuillus intolérants pourraient s'établir en raison des conditions de réchauffement et de décomposition accélérée qui libèrent une quantité importante d'éléments nutritifs (Youngblood et Titus 1996) mais un déclin surviendrait lorsque cet effet éphémère cesse et que les conditions nutritives originales se rétablissent.

Même si les coefficients de distribution sont relativement stables après 10 ans, cette variable a démontré un pouvoir prédictif limité quant au rendement à 38 ans. En ce sens, plusieurs facteurs peuvent venir influencer le développement de la régénération. Même si la taille de notre échantillon ne permet pas de tirer des conclusions fermes, certaines pistes de réflexion peuvent être avancées.

La fertilité du site influencera ainsi le lien entre l'abondance de la régénération et le rendement à maturité. Le type écologique permet de regrouper les stations présentant des caractéristiques similaires, ce qui se traduit généralement en des fertilités assez semblables (Gosselin et al. 2001). Pour les feuillus et en particulier le bouleau blanc, on remarque ainsi que les rendements pour un même coefficient de distribution tendent à être supérieurs pour les types écologiques associés à la série évolutive de la sapinière à bouleau blanc par rapport aux types associés à la sapinière à épinette noire. Le lien avec la fertilité de la station est toutefois beaucoup moins net pour le rendement en résineux. Ainsi, les types associés aux stations les moins fertiles (RE21 et RE22) ne présentent pas des rendements inférieurs pour un même coefficient de distribution. Au contraire, ils tendent à se retrouver au-dessus de la droite décrivant la relation entre le coefficient de distribution et le rendement. D'autres facteurs sont donc impliqués.

La présence d'une régénération résineuse de forte taille pourrait contribuer à l'obtention de volumes plus importants à un âge donné. Plusieurs auteurs ont en effet observé un lien direct entre un indice de diamètre ou de hauteur de la régénération et le rendement (Bertrand et al. 1992, Lussier et al. 1992, Paquin et Doucet 1992a, Tremblay et al. 1997, Laflèche et al. 2004). Bien que l'indice de diamètre ait amélioré les corrélations avec le rendement par rapport au coefficient de distribution dans le cas du sapin, de l'ensemble des feuillus et du peuplier faux-tremble, aucune amélioration n’a été constatée pour la régénération de l'épinette noire et de l'ensemble des résineux.

Le lien entre l'abondance de la régénération et le rendement pourrait aussi être influencé par la compétition interspécifique. Puisque la taille de la régénération protégée devrait influencer sa capacité de compétitionner avec une végétation feuillue installée après coupe, l'utilisation d'un indice de diamètre ou de hauteur devrait permettre l'obtention d'une meilleure prédiction du rendement. Ceci a été observé pour le sapin baumier mais pas pour l'épinette noire qui est associée à des stations moins fertiles où les feuillus sont moins agressifs. Cette compétition moins agressive sur les stations de la pessière à épinette noire et mousses pourrait expliquer que le rendement pour un même coefficient de distribution tend à y être supérieur. Malgré l'amélioration de la prédiction du rendement pour le sapin, l'utilisation de l'indice de diamètre ne permet d'expliquer que $12 \%$ de la variation du rendement.

La mortalité causée par des perturbations naturelles peut contribuer à une variation additionnelle du rendement. La région à l'étude a été affectée par deux épidémies de la tordeuse des bourgeons de l'épinette depuis la coupe, soit en 1973-77 et 1980-86. Même si les peuplements étaient jeunes à ces moments, nous avons pu constater que la majorité de la mortalité de sapin retracée correspondait à ces périodes et que des baisses de croissance importantes y étaient associées chez les tiges survivantes (Dubois 2004). La mortalité associée à l'insecte semblait faible pour l'épinette noire, même si des baisses de croissance ont été détectées. En parallèle, des accélérations de croissance ont été détectées chez les feuillus. Cet insecte constitue un élément important de la dynamique naturelle du domaine bioclimatique de la sapinière à bouleau jaune et de la bétulaie jaune à sapin. Bien que chaque étude constitue un cas particulier en termes d'intensité d'épidémie et d'âge de peuplement, nos données démontrent bien l'impact que peut avoir l'insecte sur la prédiction des rendements. Un suivi de parcelles permanentes situées dans la même région confirme l'impact majeur de l'insecte sur notre capacité de prédiction du rendement (Dubois 2004). Ainsi, dans le cas de cette dernière étude, la surface terrière du sapin à 20 ou même 40 ans ne permettait pas de prédire le rendement à 70 ans.

Lapproche dendrochronologique utilisée dans cette étude a permis de retracer l'évolution des peuplements depuis la coupe. Sarrasin (1991) affirme qu'il est possible que des événements comme la mortalité de semis ou de gaules au cours de l'évolution du peuplement ne transparaissent pas dans les résultats. Cette limite est particulièrement importante lorsque l'on tente de reconstituer la densité de la régénération ou que l'on se rapproche de la date de la coupe. Ainsi Ruel et al. (1995) ont constaté que la mortalité pouvant être importante dans les premières années après la coupe. Les tiges mortes étant relativement petites et le temps au cours duquel elles ont pu se détériorer étant plus grand, le risque de ne pas les détecter augmente lorsque l'on remonte aux premières années après la coupe. Cet effet sera davantage critique si on tente de reconstituer la densité. Une approche centrée le coefficient de distribution, incluant un effort pour retracer les tiges mortes, minimise ce problème et devrait permettre une reconstitution valide du développement du peuplement.

Bien que la problématique de l'augmentation de la composante feuillue semble présente sur le territoire à l'étude, les résultats démontrent que l'ampleur du phénomène varie selon le type de peuplement d'origine, étant particulièrement important dans les sapinières. Ceci contraste avec les observations de Laflèche et al. (2004) à l'effet que l'envahissement par les feuillus était souvent temporaire. Ainsi, malgré le fait que les sapinières se distinguent des peuplements mélangés à dominance feuillue par une distribution à dix ans en essences feuillues significativement inférieure, on constate aujourd'hui que cette composante a tout de même un impact 
majeur sur la composition des peuplements de retour. L'importante mortalité du sapin baumier engendrée par les épidémies de tordeuse des bourgeons de l'épinette serait en cause. Quant aux peuplements constitués d'épinette noire avant coupe, ils semblent se reconstituer après coupe, ce qui serait favorisé par le fait que l'épinette noire est moins vulnérable aux épidémies de tordeuse des bourgeons de l'épinette et que les conditions de croissance rencontrées dans ce type de peuplement ne sont généralement pas très favorables à l'installation des essences feuillues.

Par ailleurs, sur la base des comparaisons de compositions, malgré une présence assez importante du sapin après coupe dans cette étude, l'augmentation du sapin baumier au détriment de l'épinette noire ne semble pas préoccupante dans les pessières noires étudiées. Ceci rejoint les conclusions de Laflèche et al. (2004) à l'effet que les pessières se reconstituaient bien après coupe. Même si nous n'avons pas constaté d'effet significatif du couvert d'origine sur les variations de la présence du sapin à 38 ans, il demeure que seulement $25 \%$ des pessières ont connu une augmentation en sapin, contre plus de $60 \%$ dans le cas des peuplements mélangés. Il apparaît aussi important de dissocier les deux essences pour la prédiction du rendement des peuplements, puisque la dynamique des peuplements varie fortement en fonction des essences.

\section{Conclusion}

Les prédictions concernant la dynamique des écosystèmes après coupe sont souvent hasardeuses. Il est généralement reconnu que les écosystèmes évoluent constamment, et ce, dans des directions qui ne sont que partiellement prévisibles (Oswald 1990, Attiwill 1994, Cook 1996, Hunter 1999). Malgré ces difficultés, les résultats de l'étude ont tout de même permis de démontrer que la composition du couvert d'origine a une influence sur la strate de retour, particulièrement quand l'épinette noire est présente ou que la composante feuillue est dominante. Dans une optique de production de matière ligneuse, il s'avère cependant indispensable de mettre en parallèle les caractéristiques de régénération résineuse et feuillue pour éviter de conclure de façon précipitée au potentiel des strates au niveau de la production résineuse. En effet, les analyses portant sur la composition actuelle des peuplements permettent d'affirmer que la composante feuillue des strates en bas âge de certaines stations se maintient dans le temps et semble gagner en importance. Les épidémies de tordeuse des bourgeons de l'épinette, les caractéristiques physiques de la station et l'autécologie des espèces présentes sont également des éléments permettant d'expliquer cette dynamique forestière.

Au niveau de la prédiction des rendements, les résultats ont mis en évidence le potentiel du coefficient de distribution et de l'indice de diamètre en bas âge à prédire le rendement futur des peuplements pour les feuillus intolérants et l'épinette noire. Cependant, pour le sapin baumier, seul l'indice de diamètre s'est avéré être efficace. Il ressort donc de cette étude que la considération de la structure de la régénération doit être un facteur servant à l'estimation de la productivité en forêt mélangée. Les peuplements étudiés ont cependant subi les effets d'épidémies sévères de la tordeuse des bourgeons de l'épinette, ce qui a eu pour conséquences d'augmenter inévitablement la variabilité associée à la prédiction de l'évolution et du rendement, tel qu'observé dans l'étude.

\section{Remerciements}

Cette étude a été financée par le Ministère des ressources naturelles, de la Faune et des Parcs, la Compagnie AbitibiConsolidated, l'Association des manufacturiers de bois de sciage du Québec, le Conseil de recherche en sciences naturelles et génie du Canada et le Fonds Abitibi-Consolidated en aménagement forestier durable. Nous désirons également remercier Réjean Cantin, Maxime Camiré, Valérie Malka, Mathieu Roberge et Dominic Toupin pour leur participation aux relevés sur le terrain ainsi qu'à l'analyse des échantillons en laboratoire.

\section{Références}

Archambault, L., R.R. Gagnon, G. Pelletier, M. Chabot et L. Bélanger. 1990. Influence du drainage

et de la texture du dépôt sur la vulnérabilité du Sapin baumier et de l'Épinette blanche aux attaques de la Tordeuse des bourgeons de l'épinette. Can. J. For. Res. $20: 750-756$.

Archambault, L., J. Morisette et M. Bernier-Carbou. 1998. Forest succession over a 20 -year period

following clearcutting in balsam fir - yellow birch ecosystem of eastern Quebec, Canada. For. Ecol. Manag. 102 : 61-74.

Attiwill, P.M. 1994. The disturbance of forest ecosystems: the ecological basis for conservative management. For. Ecol. Manag. 63(2-3): 247-300.

Batzer, H.O. et M.P. Popp. 1985. Forest succession following a spruce budworm outbreak in Minnesota. For. Chron. 61 : 75-80.

Bégin, J. et F. Raulier. 1995. Comparaison de différentes approches, modèles et tailles d'échantillon pour l'établissement de relations hauteur-diamètre locales. Can. J. For. Res. 25 : 1303-1312.

Bell, F.W. 1991. Critical silvics of conifer crop species and selected competitive vegetation in northwestern Ontario. For. Can., Sault Ste. Marie, Ont. COFRDA Report 3310.

Bergeron, Y. et B. Harvey. 1997. Basing silviculture on natural ecosystem dynamics: an approach applied to the southern boreal mixedwood forest of Quebec. For. Ecol. Manag. 92(1-3): 235-242.

Berny, F. 1999. Utilisation de la procédure MIXED en présence de mesures répétées. SAS ACTA, Lettre d'information no 29. 19 p. [En ligne]. http://www.acta-informatique.fr (Page consultée le 19 mars 2003).

Bertrand, L., L. Bélanger et R.L. Beauregard. 1992. Croissance de sapinières à bouleau blanc boréales issues de coupe avec protection de la régénération. Can. J. For. Res. 22 : 1701-1711.

Boivin, J.L. 1977. Régénération après coupes mécanisées et conventionnelles : Côte-Nord du Québec. For. Chron. 53 : 341-347.

Conseil de la recherche forestière du Québec. 2002. Priorités de recherche pour le secteur forestier québécois 2002. Minist. Ressour. nat. Québec, Québec.

Cook, J.E. 1996. Implications of modern successional theory for habitat typing: a review. For. Sci. 42(1) : 67-75.

Doucet, R. 1989. Regeneration silviculture of aspen. For Chron. 65 : 23-27.

Dubois, J. 2004. Dynamique et estimation du rendement des strates de retour après coupe totale dans la sous-région écologique des collines de la rivière Vermillon. Université Laval, Mémoire de maîtrise. 110 p.

Dupont, A., L. Bélanger et J. Bousquet. 1991. Relationships between balsam fir vulnerability to spruce budworm and ecological site conditions of fir stands in central Quebec. Can. J. For. Res. 21 : 1752-1759.

Frank, R..M. 1990. Abies balsamea (L.) Mill. Balsam fir. Dans Silvics of North America: 1. Conifers. Agriculture Handbook 654. pp. 26-35. U.S. Department of Agriculture, Forest Service, Washington, DC.

Frey B.R., V.J. Lieffers, S.M. Landhausser et P.G. Comeau. 2003. An analysis of sucker regeneration of trembling aspen. Can. J. For. Res. $33: 1169-1179$. 
Frisque, G., G.F. Weetman et E. Clemmer. 1978. Analyse, 10 ans après coupe de bois à pâte, des problèmes de régénération dans l'est du Canada. Rapport technique, Report No RT-23, FERC,.

Fye, R.E. et J.B. Thomas. 1963. Regeneration of balsam fir and spruce about fifteen years following release by spruce budworm attack. For. Chron. 39 : 385-397.

Ghent, A.W., D.A. Fraser et J.B. Thomas. 1957. Studies of regeneration in forest devastated by spruce budworm. For. Sci. 3 : 184-208.

Gosselin, J., P. Grondin et J.-P. Saucier. 2001. Rapport de classification écologique de la sapinière à bouleau jaune de l'ouest (révisé). Ministère de Ressources naturelles du Québec, Direction des inventaire forestiers / Direction de la recherche forestière. $163 \mathrm{p}$. Grondin, P., C. Ansseau, L. Bélanger et coll. 1996. Écologie forestière. Dans Manuel de foresterie. pp. 133-279. Les Presses de l'Université Laval, Sainte-Foy.

Groot, A. 1996. Regeneration and surface condition trends following forest harvesting on peatlands. Northern Forestry Program, NODA/NFP tech. rep. TR-26. 12 p.

Harvey, B.D. et Y. Bergeron. 1989. Site patterns of natural regeneration following clear-cutting in north western Quebec. Can. J. For. Res. 19 : 1458-1469.

Hatcher, R.J. 1960. Croissance du sapin baumier après une coupe rase dans le Québec. Canada Ministère du nord canadien, Division des recherches sylvicoles, mémoire technique no. $87.24 \mathrm{p}$

Hibbs, D.E. 1983. Forty years of forest succession in central New England. Ecology 64 : 1394-1401.

Hunter, M.L. 1999. Maintaining biodiversity in forest ecosystems. Cambridge University Press, Cambridge, UK.

Jobidon, R. 1995. Autoécologie de quelques espèces de compétition d'importance pour la régénération forestière au Québec. Revue de littérature. Min. des Ress. nat. Québec, Québec, Mémoire rech. for. No. 117.

Kimmins, J.P. 2002. Emulation of natural forest disturbance: What does this mean, and how closely does management-induced disturbance duplicate the ecological effects of non-human forest ecosystem disturbance process? Dans L.J. Buse and A.H. Perera (Comps.) Emulating natural forest landscape disturbances: concepts and applications. pp. 15-17. Ontario Minist. Nat. Resour., Ontario For. Res. Institute, For. Res. Info. Pap. No. 149.

Kneeshaw, D. et Y. Bergeron. 1996. Ecological factors affecting the abundance of advance regeneration in Quebec's southwestern boreal forest. Can. J. For. Res. $26: 888-898$.

Laflèche, V., J. Bégin et J.-C. Ruel. 2004. Effets de la coupe avec protection de la régénération sur la production des peuplements forestiers. Mémoire de rech. for., Report 146, Min. des Ress. naturelles, de la Faune et des Parcs, Sainte-Foy.

Lussier, J-M., H. Morin et R. Gagnon. 1992. Comparaison de la croissance de marcottes d'épinette noire (Picea mariana) adultes après coupe à celle d'individus issus de graine après feu. Can. J. For. Res. 22: 1524-1535.

Meredith, M.P. et S.V. Stehman. 1991. Repeated measures experiments in forestry: focus on analysis responses curves. Can. J. For. Res. 21 : 957-965.

Ministère des Ressources Naturelles, de la Faune et des Parcs du Québec (MRNFP). 2003. Manuel d'aménagement forestier, $4^{\mathrm{e}}$ édition.

Morin, H. 1994. Dynamics of balsam fir forests in relation to spruce budworm outbreaks in the Boreal Zone of Quebec. Can. J. For. Res. $24: 730-741$

Morin, H. et R. Gagnon. 1991. Structure et croissance de peuplements d'épinette noire issus de régénération préétablie, une quarantaine d'années après coupe au Lac St-Jean, Québec. For. Chron. $67: 275-283$.

Morin, H. et R. Gagnon. 1992. Comparative growth and yield- and seed-origin black spruce (Picea mariana) stands in Quebec. Can. J. For. Res. 22 : 465-473.
Newton, P.F. 1990. Initial size hierarchy development within secondgrowth black spruce stands. Can. J. For. Res. 20 : 1720-1731.

Oliver, C.D. et B.C. Larson. 1990. Forest Stand Dynamics. McGrawHill, New York. 467 p.

Oswald, E.T. 1990. Theoretical overview of successional considerations in vegetation management. For. Chron. 66(4) : 361-365.

Paquin, R. et R. Doucet. 1992a. Productivité de pessières noires boréales régénérées par marcottage à la suite de vieilles coupes totales au Québec. Can. J. For. Res. 22 : 601-612.

Paquin, R. et R. Doucet. 1992b.Croissance en hauteur à long terme de la régénération préétablie dans des pessières noires boréales régénérées par marcottage, au Québec. Can. J. For. Res. 22 : 613-621.

Perala, D.A. 1990. Populus tremuloides Michx. Quaking aspen. Dans Silvics of North America: 2. Hardwoods. Agriculture Handbook 654. pp. 555-569. U.S. Department of Agriculture, Forest Service, Washington, DC.

Perron, J-Y. 1985. Tarif de cubage général, volume marchand brut. Ministère de l'Énergie et des Ressources, Service des inventaires forestiers. $52 \mathrm{p}$.

Pominville, P. 1993. Évolution quinquennale de la régénération protégée lors de récoltes par abattage manuel dans des sapinières. For. Chron. 69 : 569-578.

Pominville, P. et J.-C. Ruel. 1995. Effets de la coupe à blanc par bandes sur la régénération obtenue après 5 ans dans les pessières noires du Québec. Can. J. For. Res. 25 : 329-342.

Pothier, D. 1996. Évolution de la régénération après la coupe de peuplements récoltés selon différents procédés d'exploitation. For. Chron. $72: 519-527$.

Pothier, D., J. Boily et R. Doucet. 1993. Influence des caractéristiques de la régénération préexistante d'épinette noire sur la croissance des tiges individuelles. Dans C.-H. Ung (Ed.). Proceedings of International Workshop: Forest Growth Models and their uses. 18-19 Nov. 1993, Québec. pp.138-147. Natural Resources Canada., Québec.

Pothier, D., R. Doucet et J. Boily. 1995. The effect of advance regeneration height on future yield of black spruce stands. Can. J. For. Res. 25 : 536-544.

Prévost, M., V. Roy et P. Raymond. 2003. Sylviculture et régénération des forêts mixtes. Dans Actes des Colloques du Carrefour de la recherche forestière. pp. 51-58. Min. Ress. nat., Faune, Parcs Québec, Québec.

Richardson, J. 1975. Regeneration after disturbance in Newfoundland forests : patterns, problems and prescriptions. Env. Can., Can. For. Serv., Newfoundland. For. Res. Cent. Inf. Rep. N-X130. 35 p.

Riopel, M. 1999. Potentiel de croissance de la haute régénération préétablie de sapin baumier: Une analyse dendrométrique au niveau de la tige et du peuplement. Mémoire de maîtrise. Faculté de foresterie et de géomatique. Université Laval. 149 p.

Robitaille, A. et J.-P. Saucier. 1998. Paysages régionaux du Québec méridional. Ministère des Ressources naturelles du Québec, Québec. $213 \mathrm{p}$.

Ruel, J.-C. 1989. Importance de la régénération préexistante dans les forêts publiques du Québec. Ann. Sci. For. 46 : 345-359.

Ruel, J.-C. 1990. Impact de la tordeuse des bourgeons de l'épinette (Choristoneura fumiferana (Clem)) sur la régénération des sapinières après coupe à blanc, M.E.R. du Québec, direction de la recherche et développement, dir. amén. forestier.

Ruel, J.-C. 1992a. Abondance de la régénération cinq ans après la coupe à blanc mécanisée de peuplements d'épinette noire (Picea mariana (Mill) BSP). Can. J. For. Res. 22 : 1630-1638.

Ruel, J.-C. 1992b. Impact de la compétition exercée par le framboisier (Rubus idaeus L.) et les feuillus de lumière sur la croissance du sapin (Abies balsamea (L.) Mill.) en régénération. Can. J. For. Res. 22 : 1408-1416 
Ruel, J.-C., R. Doucet et J. Boily. 1995. Mortality of balsam fir and black spruce advance growth, three years after clearcutting. Can. J. For. Res. 25 : 1528-1537.

Ruel, J.-C. et M. Huot. 1993. Impact de la tordeuse des bourgeons de l'épinette (Choristoneura fumiferana (Clem)) sur la régénération des sapinières après coupe à blanc. For. Chron. 69 : 163-172.

Ruel, J., F. Ouellet, R. Plusquellec et C.H. Ung. 1998. Évolution de la régénération de peuplements résineux et mélangés au cours des 30 années après coupe à blanc mécanisée. For. Chron. 74(3): 428- 443. Sarrasin, R. 1991. Dynamique de sapinières et de pessières boréales sur une période de 40 ans après la coupe. Thèse de maîtrise, Université du Québec à Chicoutimi.

SAS Institute Inc. 1989. SAS/STATS User's guide, Version 6, Fourth Edition. SAS Institute Inc., Cary, NC.

Symons, E.S. 1996. Natural regeneration of hardwood and soft wood tree species following full-tree harvesting in northwestern Ontario. NODA/NFP Technical Report, Report TR-22, Northern Forestry Program, Natural Resources Canada, Canadian Forest Service.

Tremblay, S., J. Bégin et L. Bélanger. 1997. Productivité de sapinières boréales de seconde venue en relation avec les caractéristiques des stations. Ministère des Ressources naturelles, Direction de la recherche forestière. Mémoire de recherche forestière no $125.72 \mathrm{p}$.
Vézina, P.-É. et H. Falardeau. 1988. Le développement de peuplements de coniferes sur une période de 50 ans après la coupe. For. Chron. 64 : 329-333.

Vézina, P.-É. et A. Linteau. 1968. Growth and yield of balsam fir and black spruce in Quebec. Department of Forestry and Rural Development Canada, Forestry Branch, Information Report Q-X-2. $58 \mathrm{p}$.

Viereck, L.A. et W.F. Johnston. 1990. Picea mariana (Mill.) BSP Black spruce. Dans Silvics of North America: 1. Conifers. Agriculture Handbook 654. pp. 227-237. U.S. Department of Agriculture, Forest Service, Washington, DC.

White, A.S. 1991. The importance of different forms of regeneration to secondary succession in a Maine hardwood forest. Bull. Torrey Bot. Club 118 : 303-311.

Youngblood, A. et B. Titus. 1996. Clearcutting-a regeneration method in the boreal forest. For. Chron. $72: 31-36$. 